\title{
Joint Optimal Design and Operation of Hybrid Energy Storage Systems
}

\author{
Y. Ghiassi-Farrokhfal, Member, IEEE, C. Rosenberg, Fellow, IEEE S. Keshav, Member, IEEE, M-B. Adjaho
}

\begin{abstract}
The wide range of performance characteristics of storage technologies motivates the use of a hybrid energy storage systems (HESS) that combines the best features of multiple technologies. However, HESS design is complex, in that it involves the choice of storage technologies, the sizing of each storage element, and deciding when to charge and discharge each underlying storage element (the operating strategy. We formulate the problem of jointly optimizing the sizing and the operating strategy of an HESS that can be used for a large class of applications and storage technologies. Instead of a single set of storage element sizes, our approach determines the Pareto-optimal frontier of the sizes of the storage elements along with the corresponding optimal operating strategy. Thus, as long as the performance objective of a storage application (such as an off-grid microgrid) can be expressed as a linear combination of the underlying storage sizes, the optimal vector of storage sizes falls somewhere on this frontier. We present two case studies to illustrate our approach, demonstrating that a single storage technology is sometimes inadequate to meet application requirements, unlike an HESS designed using our approach. We also find simple, near-optimal, and practical operating strategies for these case studies, which allows us to gain several new engineering insights.
\end{abstract}

Index Terms-Smart grids, Energy storage, Hybrid storage systems, Energy efficiency, Power efficiency.

\section{INTRODUCTION}

Storage devices can enhance the performance of future smart grids in several ways; they can convert unreliable and intermittent renewable energy into a predictable and smooth source of power, encouraging and facilitating large scale renewable integration to the grid. Storage devices can be used for arbitrage; i.e., to buy electricity at low prices and sell it back when it is more expensive. Similarly, they can be used for demand shaping with the goal of reducing electricity bills. Load demands are much more controllable when storage devices are used for load leveling or peak shaving. Finally, storage devices can guarantee supply-demand matching in offgrid locations.

Each of these applications of storage has different quality requirements and performance constraints. Moreover, a wide range of storage technologies are available today, each with unique performance characteristics, but none being able to

Y. Ghiassi-Farrokhfal (y.ghiassi@rsm.nl) is with the Department of Technology and Operations Management at Rotterdam School of Management, Erasmus University and was a post-doctoral fellow at the University of Waterloo at the time of the work. S. Keshav (keshav@uwaterloo.ca) is with the Cheriton School of Computer Science, University of Waterloo. C. Rosenberg (cath@uwaterloo.ca) is with the Department of Electrical and Computer Engineering where M-B. Adjaho (mb.adjaho@telecom-bretagne.eu) was an exchange student at the time of the work.

Manuscript received July 20, 2015; revised November 7, 2015. meet the requirements of all applications. For instance, supercapacitors provide very high power, but low energy capacity, making them unsuitable for off-grid microgrids. In contrast, compressed air storage provides high energy capacity but relatively lower power, making them unsuitable for loads with a high peak-to-average power ratio. This motivates us to design hybrid energy storage systems (HESS) that combine the beneficial features of several storage elements to satisfy the performance requirements of an application [1], [2], [3]. The optimal design of an HESS must take advantage of the best features of the underlying storage elements and overcome their weaknesses.

There are at least three levels of freedom in an HESS design: (1) the choice of storage technologies, (2) the sizing of the storage elements, and (3) the charging and discharging operating strategy. The coupling among these three factors makes an optimal design complex. Existing work typically considers only one of these three factors in HESS design (e.g., [4], [5], [6]), not exploiting the full design space. Thus, the actual gain of the optimal HESS design when compared with a single-technology storage system is yet unknown (and possibly underestimated). Moreover, existing work is very applicationdependent, not applicable even with minor changes to another application [6], [7], [3], [8], [9]. Such studies consider very specific objective functions using different weights for storage unit pricing, storage lifetime and volume, or ROI. This paper is an attempt to address these shortcomings.

We study the broad class of applications that can be described in the form of the following offline demand-supply matching problem: To derive the optimal sizing and optimal operating strategy for a HESS given a particular set of storage technologies, a representative input power trace (the supply) and a corresponding committed output power trace (the demand) subject to the constraint that the demand be met at all times. Our solution returns a Pareto-optimal frontier of the sizes of the underlying storage technologies. This frontier can be used to find the optimal operating point of any application with a linear objective function that is non-decreasing in the vector of storage sizes. Note that our approach jointly optimizes the HESS size(s) and operating strategy. By repeatedly using our approach for different combinations of storage technologies, we can find the optimal design of an HESS, accounting for all three free factors.

Our framework applies to a large class of storage technologies that are characterized by a set of general evolution equations given in Section III. This class includes batteries, super capacitors (SCap), and compressed air energy storage devices (CAES) [10]. However, our approach is trace- 
dependent and assumes that the entire trace is known ahead of time. This is typical for a design problem (e.g., the sizing of the elements) but impractical for designing a useful operating strategy. Nevertheless, the trace-dependent optimal operating strategy can serve as the benchmark for any practical operating strategy (i.e., a rule-based strategy that is myopic). Indeed, in this paper, we present practical operating strategies inspired by the offline optimal. Specifically, we use our framework for two case studies: solar power firming and self-usage in off-grid scenarios and show that there are simple, practical, near-optimal operating strategies.

Our key contributions are:

- We develop a joint HESS optimal sizing and operating strategy formulation for a large class of supply-demand matching applications and a broad class of storage elements. We further convert it to a linear programming (LP), substantially reducing the solution time. Our formulation returns a Pareto-frontier of the minimum sizes of the storage elements. This frontier can be used to find the optimal storage sizes of different applications depending on their objective functions.

- We construct a simple RC-based energy evolution model for supercapacitors and show that it has the same generic form as the energy evolution model of batteries.

- We apply our formulation to two important case studies and find near-optimal practical operating strategies for each case.

- We use real-world power traces for these applications and provide numerical results, which lead to several interesting engineering insights. For example, we show that a single storage technology is sometimes inadequate to provide the requirements of the storage application, while a well-designed HESS is adequate.

The rest of the paper is structured as follows. In Section II, we discuss the main characteristics of storage technologies, motivate the use of an HESS, and introduce our system model. We formulate our offline design problem in Section III. Then, we describe the two case studies in details in Section IV. In Section V, we provide some numerical examples for our cases studies. We explore the related work in Section V and conclude the paper in Section VII.

\section{SySTEM DEsCRIPTION AND MOdeL}

\section{A. System description and characteristics of storage}

There are several storage technologies such as mechanical, thermo-dynamic, electrochemical, and electro-magnetic, each with its own operating characteristics [10], [8]. Depending of the applications, some of these operating characteristics could be a bottleneck. For example, due to inherent inefficiencies, each unit of energy stored is reduced by a certain efficiency factor when added to (or discharged from) a storage device (the charge (discharge) efficiency). As another example, stored energy leaks over time (self-discharge). The leak rate can be a function of several parameters such as state of charge, storage size, and temperature, depending on the storage technology. In addition, there is a limit on the maximum charge (discharge) power (the maximum charge (discharge) rate). Finally, one of

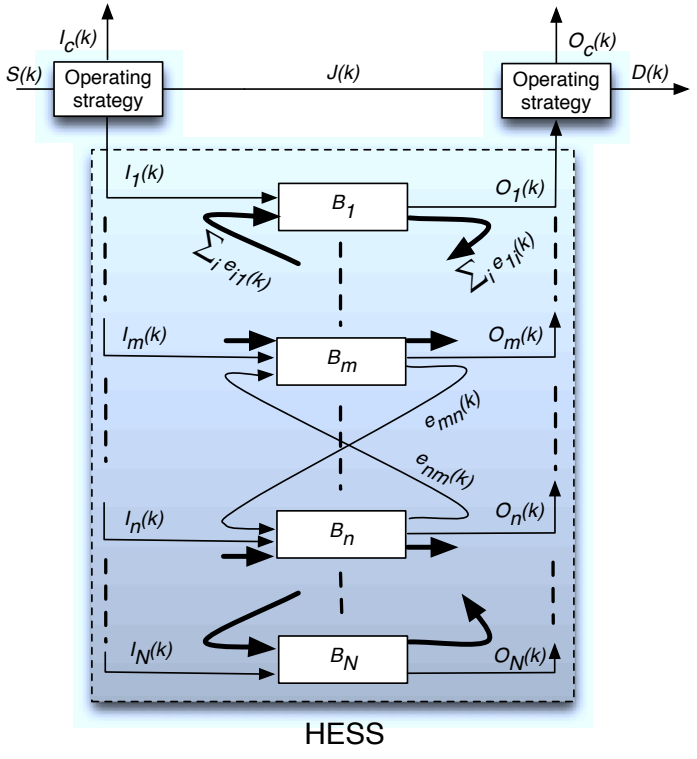

Fig. 1: System model

the most important characteristics of storage systems is their energy (resp. power) density. Energy (resp. power) density is the maximum amount of energy (resp. power) that can be stored in (resp. drawn in or out of) a given storage device per unit volume.

Most storage imperfections are not an issue if storage can be infinitely sized. The size of storage is, however, severely limited due to the high prices of the current storage technologies. The cost is typically presented either per unit of energy or per unit of power, depending on the requirements of the application.

Typically, a storage device has some mix of good and poor operating characteristics ${ }^{1}$. For example, there is no single technology which is both energy efficient and power efficient. Well-designed HESS can possibly exploit the best features of its underlying storage technologies, while hiding their weaknesses.

\section{B. System model}

Our supply-demand matching system model is described in Fig. 1. We assume that the HESS is composed of $N$ storage devices. We consider a discrete time model; i.e., $t=0, \delta, 2 \delta, \ldots$, where $\delta$ is the time unit and a time horizon $T=K \delta$ (typically a year). We assume that all variables remain constant in a time-slot. A supply power $S(k)$ is the input to the HESS that commits to provide the given committed output power $D(k)$ at any time $k$. For the offline design problem, we assume that we have representative traces for both $S(k)$ and $D(k)$ over the horizon $T$. The goal is to optimally design such an HESS, accounting for all possible choices of the three levels of freedom: the operating strategy, the choice of storage technologies, and storage sizing.

Operating strategies determine three actions at any time, given the input and the current state of the system (i.e., input

\footnotetext{
${ }^{1}$ To date, there is no single storage technology which outperforms others in all aspects.
} 


\begin{tabular}{|c|c|}
\hline Name & Description (unit) \\
\hline$S(k)$ & The input power in time slot $k(W)$ \\
\hline$D(k)$ & The committed output power in time slot $k(W)$ \\
\hline$r$ & The ratio of the long-term average of output to the long-term average of the input $(0 \leq r \leq 1)$ \\
\hline$J(k)$ & The output power directly from source in time slot $k(W)$ \\
\hline$I_{i}(k)$ & The input power put into storage $i$ at $k(W)$ \\
\hline$I_{c}(k)$ & The curtailed input power at $k(W)$ \\
\hline$O_{c}(k)$ & The curtailed output power at $k(W)$ \\
\hline$O_{i}(k)$ & The output power drawn from storage $i$ at $k(W)$ \\
\hline$e_{i j}(k)$ & The power migration from storage $i$ to storage $j$ at $k(W)$ \\
\hline$b_{i}(k)$ & The energy content of storage $i$ at $k(W h)$ \\
\hline$a_{i}$ & Maximum usable fraction of storage $i$ \\
\hline$\Gamma_{i}$ & The self-discharge ratio of storage $i$ \\
\hline$\gamma_{i}$ & The self-discharge energy of storage $i(W h)$ \\
\hline$\eta_{i}^{c}$ & The charging efficiency of storage $i$ \\
\hline$\eta_{i}^{d}$ & The discharging efficiency of storage $i$ \\
\hline$\alpha_{i}^{c}$ & The charging rate of storage $i$ (in $\left.h r^{-1}\right)$ \\
\hline$\alpha_{i}^{d}$ & The discharging rate of storage $i$ (in $\left.h r^{-1}\right)$ \\
\hline$B_{i}$ & The size of storage $i(W h)$ \\
\hline$N$ & Total number of storage devices in HESS \\
\hline$T$ & Time horizon (in $h)$ \\
\hline$K$ & Time horizon in number of time slots \\
\hline$\delta$ & Time unit (in $h$ ) \\
\hline
\end{tabular}

TABLE I: List of Notations.

power, output power, and the state of charge of the storage elements): charge (what is the charging power to each element of the HESS?), discharge (what is the discharging power from each element of the HESS?), and charge migration: (what is the energy that must be transferred from one storage device to the other?)

Power traces are time series vectors with the $k$ 'th element corresponding to the value of that element at time $k \delta$. The input power $S(k)$ at time $k$ can be (possibly partly) delivered directly to the output (denoted by $J(k)$ ) or be (partly) stored at some of the $N$ storage devices (the corresponding charging being denoted by $I_{i}(k)$ for any $\left.i \in\{1, \ldots, N\}\right)$, or undesirably curtailed $\left(I_{c}(k)\right)$. Each storage device $i$ can also migrate energy at any time slot $k$ to another device $j$ with power $e_{i j}(k)$. To simplify notation, we also define self-migration and we set it to zero $\left(e_{i i}(k)=0\right.$ for any $i$ and $k$ ). Finally, each storage device $i$ can be discharged to serve the output power at any time slot $k$ (denoted by $O_{i}(k)$ for any $\left.i \in\{1, \ldots, N\}\right)$ and the surplus output power $O_{c}(k)$ at any time $k$ is discarded. Please see Table I for a list of notations. Designing an operating strategy corresponds to selecting appropriate values for $\left(O_{i}(k), e_{i j}(k), I_{i}(k), J(k)\right.$, $I_{c}(k), O_{c}(k)$ for $\left.i, j \in\{1, \ldots, N\}\right)$.

Our system model in Fig. 1 enforces some constraints on these variables: Firstly, the net input and output powers at the HESS connecting points to the system must be zero; i.e.,

$$
\begin{array}{ll}
I_{c}(k)+J(k)-S(k)+\sum_{i=1}^{N} I_{i}(k)=0 & \forall k \\
J(k)-D(k)-O_{c}(k)+\sum_{i=1}^{N} O_{i}(k)=0 & \forall k
\end{array}
$$

Secondly, all variables are non-negative. We can treat $I_{c}(k)$ and $O_{c}(k)$ as slack variables and remove them from the list of independent variables and replace $I_{c}(k), O_{c}(k) \geq 0$ and
Eqs. (1), (2) equivalently with

$$
\begin{array}{ll}
\sum_{i=1}^{N} I_{i}(k) \leq S(k)-J(k) & \forall k \\
J(k)+\sum_{i=1}^{N} O_{i}(k) \geq D(k) & \forall k
\end{array}
$$

We assume that the storage devices in our framework belong to the broad class of storage whose operation can be described as the following (applied to storage device $i$ ) [11], [12].

$$
b_{i}(k)=\Gamma_{i} b_{i}(k-1)+\eta_{i}^{c} c_{i}(k) \delta-\frac{1}{\eta_{i}^{d}} d_{i}(k) \delta-\gamma_{i} \quad \forall k
$$

where $c_{i}(k)$ (resp. $\left.d_{i}(k)\right)$ is the charging (resp. discharging) power in time slot $k . c_{i}(k), d_{i}(k)$, and $b_{i}(k)$ must satisfy for any $k$ :

$$
\begin{aligned}
& 0 \leq c_{i}(k) \leq \alpha_{i}^{c} B_{i} \\
& 0 \leq d_{i}(k) \leq \alpha_{i}^{d} B_{i} \\
& c_{i}(k) d_{i}(k)=0 \\
& 0 \leq b_{i}(k) \leq a_{i} B_{i}
\end{aligned}
$$

In words, the storage device $i$ cannot be charged (discharged) faster than $\alpha_{i}^{c} B_{i}\left(\alpha_{i}^{d} B_{i}\right)$ Watts, where $B_{i}$ is the size of the storage device $i$. A fraction $1-\eta_{i}^{c}\left(1-\eta_{i}^{d}\right)$ of the total energy being charged to (withdrawn from) the storage device $i$ is lost due to energy conversion inefficiency. The storage device $i$ operates properly (i.e., with an appropriate lifetime) if only a fraction $a_{i}$ of the entire storage device is used. The storage cannot charge and discharge at the same time and finally, the stored energy leaks over time by as much as $\left(1-\Gamma_{i}\right) b_{i}(k)+\gamma_{i}$ during time slot $k$, where $b_{i}(k)$ is the energy stored in element $i$ at time $k$. 
Note that in the system described in Fig. 1, we have for all $i$ and $k$ :

$$
\begin{aligned}
& c_{i}(k)=I_{i}(k)+\sum_{j=1}^{N} e_{j i}(k) \\
& d_{i}(k)=O_{i}(k)+\sum_{j=1}^{N} e_{i j}(k)
\end{aligned}
$$

\section{Pareto-optimal Sizing of AN HESS}

The objective of our offline optimization problem is to minimally size the elements of the HESS, given representative traces for $S$ and $D$, such that $D$ is always met (note that this problem might not always be feasible). We jointly optimize the vector of storage sizes $\left(B_{1}, B_{2}, \ldots, B_{N}\right)$ and the operating strategy; i.e., the variables $\left(O_{i}(k), e_{i j}(k), I_{i}(k), J(k)\right)$ for any $i, j \in\{1, \ldots, N\}$ and any $k \in\{0, \ldots, K\}$. Specifically, using the above definitions and notation and given $(S(k)),(D(k))$, the parameters of the storage elements $\left(\alpha_{i}^{c}, \alpha_{i}^{d}, \eta_{i}^{c}, \eta_{i}^{d}, a_{i}\right.$, $\gamma_{i}$, and $\Gamma_{i}$ for any $\left.i \in\{1, \ldots, N\}\right)$, we want to solve the following multi-objective problem to find the Pareto-optimal frontier $\left(B_{1}, B_{2}, \ldots, B_{N}\right)$ :

$$
\underset{\substack{\left(B_{i}\right),\left(O_{i}(k)\right),\left(I_{i}(k)\right),(J(k)),\left(e_{j i}(k)\right)}}{\operatorname{Minimize}}\left(B_{1}, \ldots, B_{N}\right)
$$

subject to

$$
\begin{aligned}
& B_{i} \geq 0 \\
& \forall i \\
& J(k) \geq D(k)-\sum_{i=1}^{N} O_{i}(k), \\
& \forall k \\
& \sum_{i=1}^{N} I_{i}(k) \leq S(k)-J(k) \\
& \forall k \\
& I_{i}(k)+\sum_{j=1}^{N} e_{j i}(k) \leq \alpha_{i}^{c} B_{i} \\
& \forall k ; \forall i \\
& O_{i}(k)+\sum_{j=1}^{N} e_{j i}(k) \leq \alpha_{i}^{d} B_{i} \\
& \forall k ; \forall i \\
& 0 \leq b_{i}(k) \leq a_{i} B_{i}, \\
& \forall k ; \forall i \\
& b_{i}(k)=\Gamma_{i} b_{i}(k-1)+\eta_{i}^{c}\left(I_{i}(k)+\sum_{j=1}^{N} e_{j i}(k)\right) \delta \\
& -\left(O_{i}(k)+\sum_{j=1}^{N} e_{i j}(k)\right) \delta / \eta_{i}^{d}-\gamma_{j} \quad \forall k ; \forall i \\
& I_{i}(k), I_{i}(k), O_{i}(k), O_{i}(k) \geq 0 \quad \forall k ; \forall i \\
& e_{i j}(k), J(k) \geq 0 \\
& \forall k ; \forall i, j(12 \mathrm{j}) \\
& \left(I_{i}(k)+\sum_{j=1}^{N} e_{j i}(k)\right) \times\left(O_{i}(k)+\sum_{j=1}^{N} e_{i j}(k)\right)=0 \forall k ; \forall i
\end{aligned}
$$

This problem can be solved iteratively by replacing the multi-objective function by the following scalarized objective:

$$
\underset{\substack{\left(B_{i}\right),\left(O_{i}(k)\right),\left(I_{i}(k)\right),(J(k)),\left(e_{j i}(k)\right)}}{\operatorname{Minimize}} \quad \sum_{i=1}^{N} w_{i} B_{i}
$$

for all weights $w_{i} \geq 0$ such that $\sum_{i=1}^{N} w_{i}=1$. Assuming a small set of underlying storage technologies (i.e, small $N$ ) and a discrete set of weights for each technology, it is possible to explore the entire state space as long as the core optimization problem can be quickly solved, i.e., is linear. However, the scalarized optimization problem is non-linear, because of the last non-linear constraint (Eq. (12k)) that is used to prevent storage elements from simultaneously charging and discharging. The following lemma shows that this inequality can be removed, converting the problem to a linear program (LP) and facilitating the use of fast LP solvers.

Lemma 1. There is an optimal solution that does not require simultaneously charging and discharging any storage element in the HESS.

Please see the Appendix A.1 for the proof.

Let us consider P1, the original NL problem with Eq. (12k) and the linear problem P2 corresponding to P1 without Eq. (12k). P2 has a larger feasibility region than $\mathrm{P} 1$. We show in Appendix A.1 that an optimal solution of P2 can always be transformed into another optimal solution of P2 where no simultaneous charging or discharging of any storage element occur. We show that this transformed optimal solution is also an optimal solution for $\mathrm{P} 1$, the original problem.

\section{Case Studies}

Recall that the Pareto sizing frontier describes the minimal set of HESS sizes necessary to always match supply and demand. For a given application, whose performance objective — such as minimizing price, volume, or weight—can be expressed as a linear combination of the storage sizes, we can find the corresponding optimal vector of HESS sizes by evaluating the application's objective only along the Pareto frontier, instead of the entire space. In this section, we apply this approach to two specific case studies. For both case studies, we consider an HESS with two storage devices (i.e., $N=2$ ). We first justify our choice of storage technologies, then discuss the two applications. This section ends with a discussion on practical operating strategies.

\section{A. Choice of Storage Technologies}

Batteries and supercapacitors are, respectively, known to be energy efficient and power efficient energy storage technologies. Thus, a combination of a battery and a SCap is an $a$ priori good combination to meet both the energy efficiency and the power efficiency requirements of an application. The energy-content evolution of these two technologies can be modelled by Eqs. (5)-(9). In the following, we assume that the first storage element (i.e., $i=1$ ) is a battery and the second (i.e., $i=2$ ) is a SCap. We now describe and characterize SCap and batteries in more detail.

\section{A.1 Batteries}




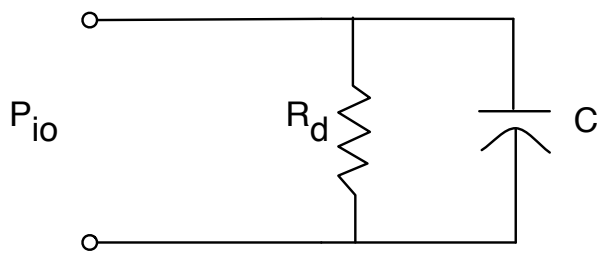

Fig. 2: SCap circuit model.

There is a large range of battery technologies; widely-used technologies include:

- Lead-acid (PbA) battery: PbA batteries are attractive due to their low up-front cost. However, their lifetime is limited and they must be frequently replaced.

- Lithium-ion (Li-ion) battery: Lithium-ion cells are typically rated for much higher charge and discharge power than $\mathrm{PbA}$ cells, making them better suited to absorb high pulses of generated power to meet large power demands. This type of battery does not require full recharge to prevent degradation, meaning that the stress of infrequent full charges found in renewable-energy applications may actually be a benefit. Finally, Lithium-ion batteries have much longer cycle lives than PbA batteries.

- NiCd battery: The most important feature of NiCd cells is that they have the highest power density among the existing batteries, making them suitable for applications with high current drain requirements. However, cadmium is a highly toxic heavy metal that can damage the environment if not disposed properly. NiCd cells suffer from so-called 'memory loss', which means that they gradually lose their maximum energy capacity if they are repeatedly recharged after being only partially discharged.

The most important imperfections of a battery are its charging/discharging inefficiencies and the limits on its maximum charging/discharging power rates [10]. Batteries have negligible self-discharge. Thus,

$$
\Gamma_{1} \approx 1 ; \quad \gamma_{1} \approx 0
$$

\section{A.2 Supercapacitors (SCap)}

SCaps have a high power density. They can be fully charged or discharged within seconds [13], which is much faster than any battery technology [14]. The cycle life of a SCap is very high (500,000 to $1,000,000$ charging cycles) compared to a battery (few thousands) [14]. However, SCap is an expensive technology ( $5 \mathrm{x}$ to $20 \mathrm{x}$ more expensive than Li-ion batteries), its energy density is low, and its self-discharge rate is quite high [14].

There are several circuit models proposed in the literature to model the operation of SCaps. When SCaps are charged and discharged within the period of a day or so, as is the case in our two applications, the simple parallel RC circuit model shown in Fig. 2 is found to be adequate. In Appendix A. 2 we show that the evolution of the energy stored in a SCap can be

\begin{tabular}{|c||c|c|c|c|}
\hline & PbA & Li-ion & NiCd & SCap \\
\hline \hline$\eta^{c} \eta^{d}$ & 0.75 & 0.9 & 0.8 & 1 \\
\hline$\alpha^{c}$ & 0.25 & 1 & 2 & large \\
\hline$\alpha^{d}$ & 2 & 2 & 20 & large \\
\hline$a$ & 0.80 & 0.80 & 0.80 & 1 \\
\hline$\Gamma$ & 1 & 1 & 1 & 0.9987 \\
\hline$\gamma$ & 0 & 0 & 0 & 0 \\
\hline
\end{tabular}

TABLE II: Storage systems characteristics in room temperature and for renewable energy applications [15], [16], [14], [8].

modelled by Eqs. (5)-(9) with

$$
\begin{aligned}
& \Gamma_{2}=\left(1-\frac{2 \delta}{R C}\right) ; \quad \gamma_{2}=0 \\
& B_{2}=1 / 2 C V_{\text {max }}^{2} ; \quad \alpha_{2}^{c}=\alpha_{2}^{d}=\infty ; \quad a_{2}=1
\end{aligned}
$$

where $C, R$, and $V_{\max }$ are, respectively, the values of the parallel capacitance, the parallel resistor, and the maximum allowable terminal voltage of the equivalent circuit model. These parameters can be easily obtained from the data sheet of an SCap.

\section{B. Applications}

Our problem formulation can be applied to any storage application for which we have representative traces for the input power $S$ and the committed output power $D$.

\section{B.1 Application 1: Firming Solar Power}

Solar power is inherently an intermittent source of power, making it unreliable. However, it can be converted to a reliable and smooth power source, using an HESS. Precisely, in this application, the solar power trace $(S(k))$ is given and we compute for each day how much we commit in terms of output power using a given parameter $r$. Hence the output power $D(k)$ is constant over a day, depends on the input power, and is computed as:

$$
D(k)=r \times \text { average of } S \text { in day } d \quad \forall k \text { in day } d
$$

Given $(S(k)$ ), the larger $r$, the larger the payoff (since we sell more power), but the larger the HESS needs to be. One can consider finding the optimal value of $r$ for different objective metrics for example, the return on investment (ROI) of the system. In this paper, however, we consider $r$ as an input to our problem. Note that $r$ does not change on a daily basis and that $0<r<1$ since the average demand cannot be larger than the average supply.

The HESS in this application has two main roles: 1) to carry over energy to be used during the night; 2) to smooth out power generation in short time scales and convert it into a constant output power. Due to high energy density of battery technologies, they are great to meet the first goal. The high power density of SCaps makes them ideal for the second goal.

\section{B.2 Application 2: Self-usage}

Another important application of storage is in off-grid scenarios. Consider a household which has solar PV panels on the roof and is not connected to the grid. The entire load demand of the household must be met by the only available source of power, which is solar power. The HESS in this 
application has two main roles: 1) to carry over energy for as long as necessary, perhaps even a few days, to ensure that there exists enough energy to meet the load demand even during several consecutive days with high load demand; 2) to guarantee that the supply-demand matching is guaranteed in all time scales. Again, battery and SCap are, respectively, good candidates to meet the first and the second goal, due to their energy and power density characteristics.

In this application, we are given an irradiance trace $E(k)$ for the location of the home. Let $\kappa$ be the efficiency of a unitarea $\mathrm{PV}$ panel and $\bar{E}$ be the long-term average of $E$. Then, $n=\frac{\bar{D}}{\kappa \bar{E}}$ is the minimum number of unit-area panels needed to make sure that the total energy produced is larger that the total energy needed. We can then choose $r$ so that the number of panels is $\frac{n}{r}$ with $0<r<1$ and then, the larger $r$, the less investment on solar PV panels, which implies more investment on HESS to meet the load demand. One can consider designing $r$ for different objective metrics such as minimizing the overall budget for the system (solar panels and HESS). In this paper, however, we consider $r$ as an input to our problem.

\section{Practical Operating Strategies}

The Pareto-optimal sizing frontier is obtained by simultaneously optimizing storage sizes along with the operation strategy. However, this optimal operation strategy is tracedependent and requires the entire supply and load traces in advance. In practice, however, this information is not available. Nevertheless, the Pareto-optimal frontier provides a benchmark for any practical operating strategy. This raises the question of how well a practical operating strategy can perform compared to the Pareto-optimal benchmark.

We define a practical strategy to be one that does not require any knowledge of the future. To design our practical operating strategies, we observe that in our numerical examples the problem defined by Eqs. (12a)-(12k), in which no migration of energy allowed, that is, $e_{i j}(k)=0 \forall i, j$ and $\forall k$, results in nearly-optimal solutions. This motivates the following two simple practical operating strategies:

Strategy 1: The SCap is used as the primary storage device for both charging and discharging. The battery is used only if the SCap fills up when it is charging or becomes empty when it is discharging.

Strategy 2: The SCap is used as the primary storage device for discharging and the battery is used as the primary storage device for charging. The battery is used for discharging only if the SCap becomes empty and we use the SCap for charging only if the battery becomes full.

Both of these operating strategies use the SCap as the primary storage for discharging. This is because, given the high self-discharge rate of SCaps, we want to minimize the time that we keep energy in SCaps. The difference between Strategy 1 and Strategy 2 is in the choice of the primary storage for charging. The relative performance of these two operating strategies depends mainly on their relative charge/discharge efficiencies and degree of self-discharge, as explained next.

Suppose there is one unit of energy that needs to be stored in an HESS. We have the option to store it either in the SCap or in the battery. Say that this energy unit with the optimal operating strategy will be withdrawn after $k_{d}$ time slots. This means that this energy unit spends $k_{d}$ time units in the storage device before it departs the device. If that energy unit is stored in the battery, by the end of $k_{d}$ time slots, we can withdraw only a fraction of $\eta_{1}^{c} \eta_{1}^{d}<1$ from that energy unit, due to the battery charging/discharging inefficiencies since there is no leakage (from Eq. (14)). If it is stored in the SCap, we can withdraw only a fraction of $\Gamma_{2}^{k_{d}}<1$ from that energy unit after $k_{d}$ time units, due to the SCap self-discharge (from Eq. (15)). Thus, the primary storage device for charging operation at any point depends on how long that energy unit is supposed to be kept in the storage device before it departs. In a myopic strategy, $k_{d}$ is not known ahead of time, and hence the optimal choice of storage for charging at any time is not known either. As a result, we propose Strategy 1 and Strategy 2. We will compare the performance of such static operating strategies with the optimal operating strategy, using real world traces in the next section.

\section{Numerical EVAluATion}

In this section, we present some numerical examples for the two case studies described in Section IV. The characteristics of the storage devices used in this section are listed in Table $\mathrm{II}^{2}$. We use the scalarized version of the optimization problem formulated in Eq. (12a) to Eq. (12j) to find the Pareto frontier and the optimal operating strategy. We also compute the minimum $B_{2}$ (given $B_{1}$ ), assuming one of the two practical operating strategies described in Section IV-C. For the source power trace, we use one year of the solar irradiance dataset from the atmospheric radiation measurement website [17] from the $\mathrm{C} 1$ station in the Southern Great Plains permanent site. For the first case study, we consider one panel and study several values for $r$ between 0 and 1. For the second case study, we use for the load demand data, anonymized hourly home-level load data provided to us by a Local Distribution Company in Ontario, Canada and scale the irradiance set by the factor $\frac{n}{r}$ as discussed above where $0<r<1$ is given. Fig. 3 helps better visualize the statistical properties of the input power and the committed output for these two applications, by plotting several daily sample paths of one month of these processes.

\section{A. Pareto-optimal Sizing}

Fig. 4 illustrates the Pareto-optimal sizing frontier of a (SCap+battery) HESS when the operating strategy is optimal for the two applications under study, three battery technologies, and several values of $r$. Note that these curves essentially show insights into HESS feasibility. For example, for the solar firming application, for $r=0.7$, the three battery technologies yield the same Pareto frontier. Moreover, since the frontiers

\footnotetext{
${ }^{2}$ Note that the imperfection parameters of a battery can be very different from its nominal values when the battery is used to store energy from renewable energy sources. In fact, the charging and discharging rates can exceed the rated values at times. The intermittent charging-discharging nature of storage operation in renewable energy applications tends to increase the efficiency of storage operation, allowing larger charging-discharging values.
} 


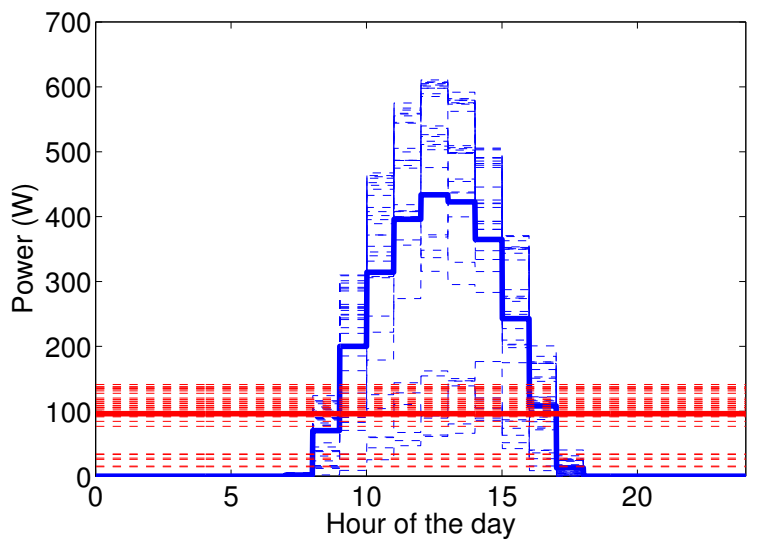

(a) Solar power firming application

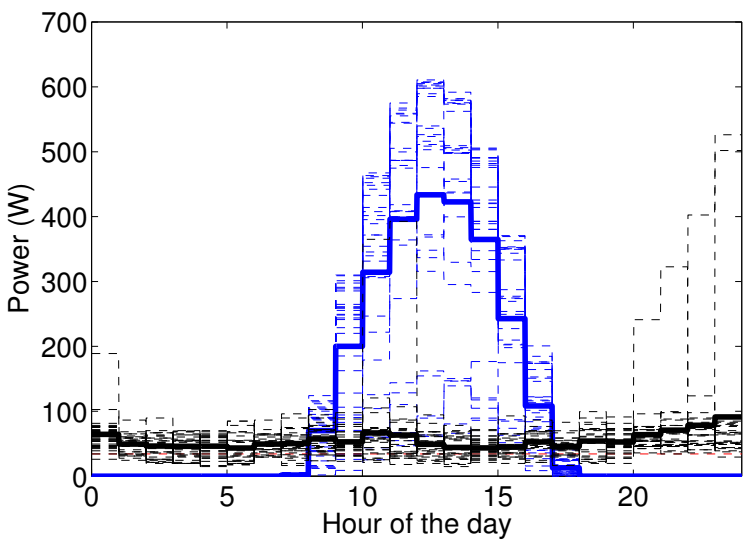

(b) Self-usage application

Fig. 3: Daily sample paths of one month of the available solar power (in blue), the committed output power for the solar power firming application (in red), and the committed output power for the self-usage application (in black). Thick curves present the average values over all the sample paths with the same colour.

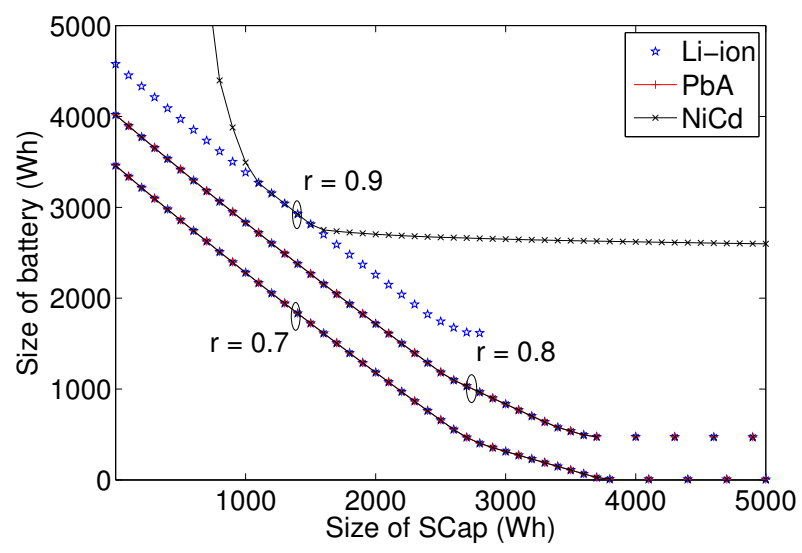

(a) Solar power firming application

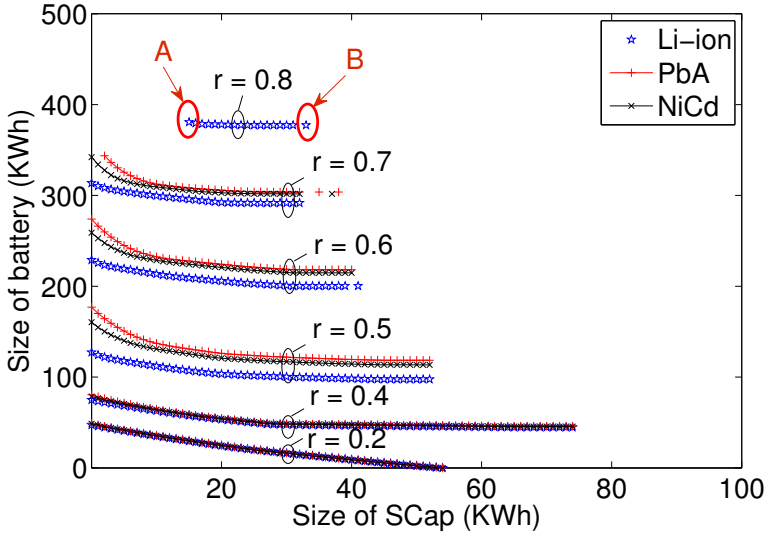

(b) Self-usage application

Fig. 4: Pareto-optimal frontier of (Scap+battery), using three battery technologies for different values of $r$.

intersect both the $\mathrm{X}$ and $\mathrm{Y}$ axes, all the batteries and SCap could also have performed solar firming by themselves. However, even though the three battery technologies yield the same Pareto frontier, it does not mean that the systems are equivalent since once an objective function based on cost has been chosen they might yield very different operating points on the frontier and one of these operating points might dominate the others. For $r=0.8$, the three battery technologies yield the same Pareto frontier and although the batteries could have performed solar firming by themselves, SCap alone could not. For $r=0.9$, an HESS with $\mathrm{PbA}$ cannot performed solar firming at all while an HESS with $\mathrm{Li}$-ion or $\mathrm{NiCd}$ can and $\mathrm{Li}$-ion seems to do it better (though again the final comparison would depend on the cost). Li-ion can perform solar firming alone while $\mathrm{NiCd}$ and $\mathrm{PbA}$ cannot.

For the self-usage application, Fig. 4 clearly shows that the larger the $r$, the more expensive the HESS is (in terms of storage sizing) and hence the more pronounced the role of the storage technology. Comparing the performance of the battery technologies and their corresponding physical imperfections in Fig. 4 suggests that the charging/discharging efficiency is the key feature that differentiates their performance.

Fig. 4b illustrates the Pareto-optimal frontier for the selfusage application. SCap alone cannot meet the load demand for $r>0.2$ while the three battery technologies can up to $r<0.8$. For $r=0.8$, the load demand cannot be met by a single storage technology and we need to have a battery of size at least $377 \mathrm{Wh}$ (denoted by point B) and a SCap of at least $15 \mathrm{KWh}$ (denoted by point $\mathrm{A}$ ) in any feasible HESS. This is because, at this large value of $r$, the battery inefficiency becomes a bottleneck and we need to combine the ideal efficiency of SCaps and the negligible self-discharge of the batteries to meet the load demand.

\section{B. The Performance of the Practical Operating Strategies}

Fig. 5 compares the performance of the proposed practical operating strategies with the optimal trace-based one for a SCap+Li-ion system for the two applications. We make several 


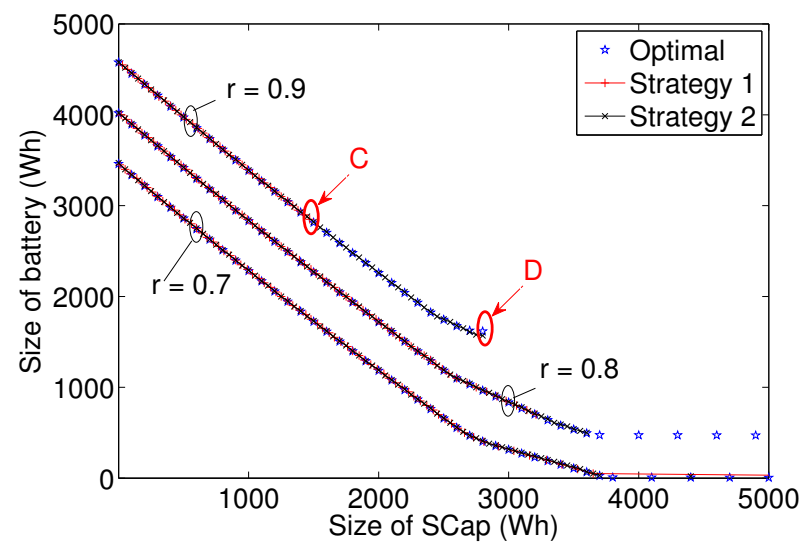

(a) Solar power firming application

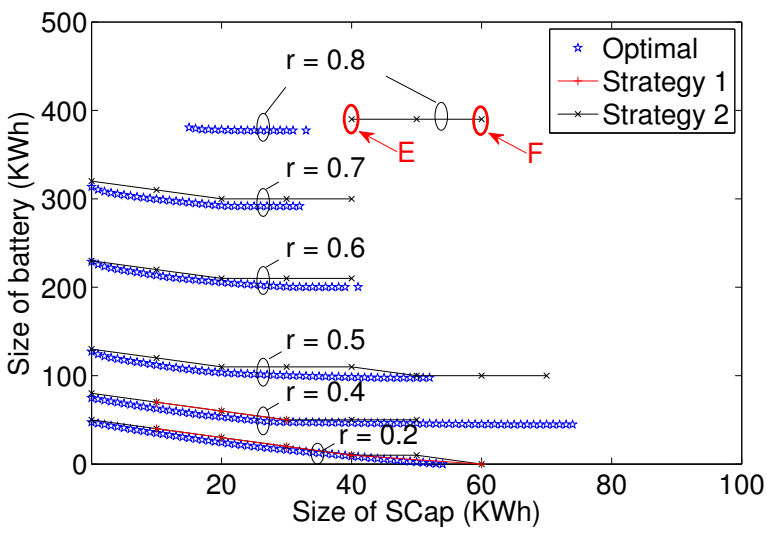

(b) Self-usage application

Fig. 5: Comparing practical operating strategies with the trace-based optimal one for a (Scap+Li-ion) HESS for different values of $r$.

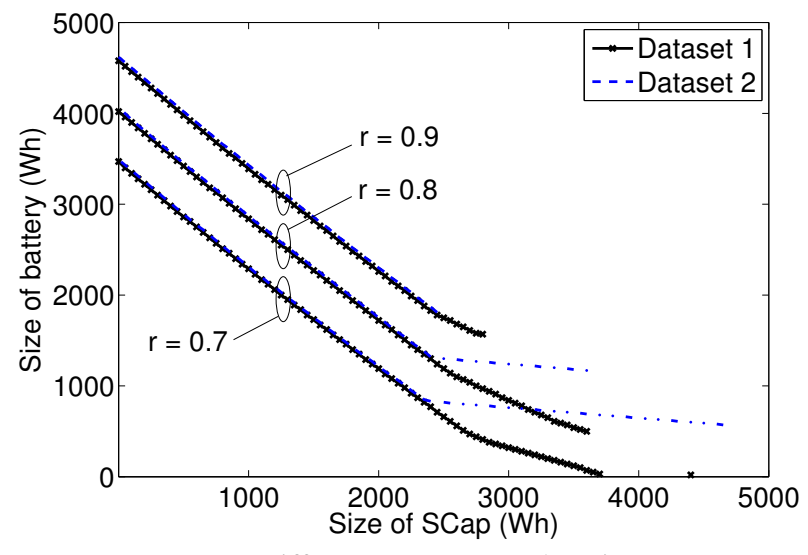

(a) Different years, same location

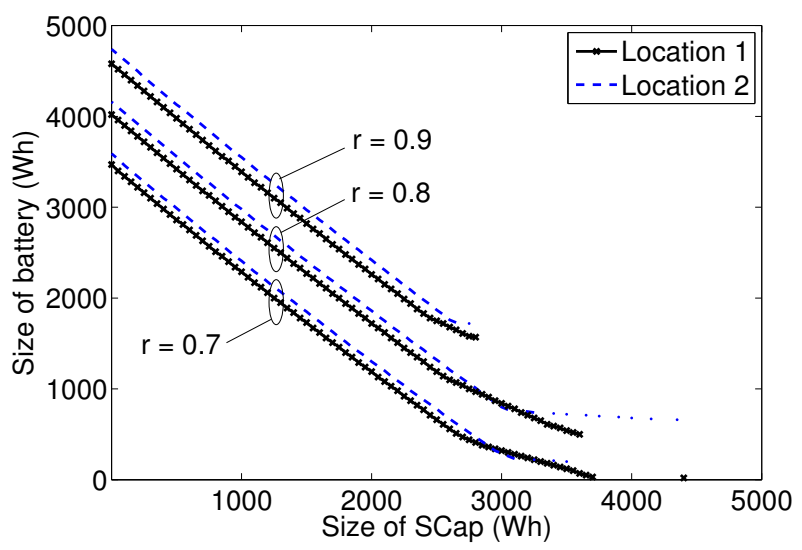

(b) Different locations

Fig. 6: Sensitivity analysis: Solar power firming application

observations. Most importantly, Fig. 5 suggests that there exists a simple myopic operating strategy (i.e., Strategy 2), which closely follows the optimal Strategy. In most cases, Strategy 2 outperforms Strategy 1 (compare points C and D, respectively, corresponding to Strategies 1 and 2 in Fig. 5a).

As explained in Section IV-C, the only difference between these two operating strategies is the choice of primary storage for charging. The better performance of Strategy 2 compared to Strategy 1 indicates that in critical scenarios, the stored energy remains in the storage device for a long time, and hence, the self-discharge of SCap is more restrictive than the battery inefficiency.

The performance of the operating strategies is also highly affected by the application. Strategy 1 becomes infeasible for any $r>0.5$ in the self-usage application. Moreover, the difference between Strategy 2 and the optimal strategy gradually increases as $r$ increases (for example, check points $\mathrm{E}$ and $\mathrm{F}$ in Fig. 5b).

\section{Sensitivity Analysis with Respect to the Input Solar Power}

We now study the sensitivity of our conclusions with respect to the input solar power for both applications. We perform a sensitivity analysis with respect to the variations of solar power in the same location from one year to another (temporal variations) and to the variations at different locations (spatial variations).

Fig. 6a compares the frontier obtained with the solar power trace used in the previous examples (Dataset 1), with a solar power trace from the same location but a different year (Dataset 2). Fig. 6b studies the sensitivity of the results with respect to the spatial variations by comparing the Paretooptimal frontier from two different sites far apart from each other. We use operating Strategy 2 for all cases. As shown in Fig. 6, the Pareto-optimal frontier for the solar firming application is almost insensitive to temporal variations and is more sensitive to spatial variations. This might stem from the fact that the output power in this application is highly correlated with the input power, making the input variability less significant.

We next do a sensitivity analysis for the self-usage appli- 


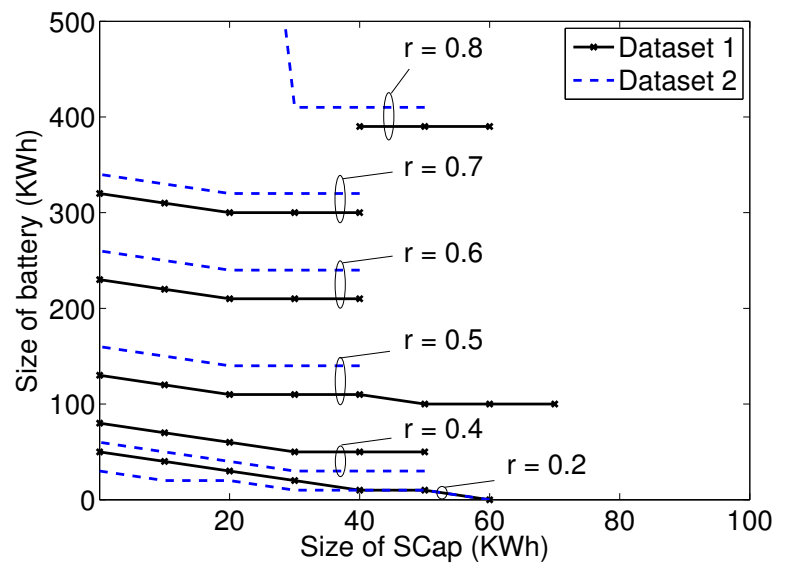

(a) Different years, same location

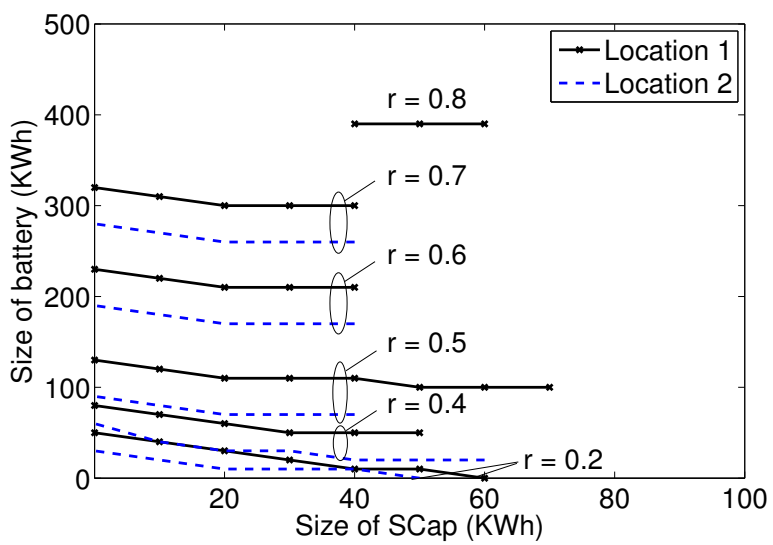

(b) Different locations

Fig. 7: Sensitivity analysis: Self-usage application

cation. Fig. 7a compares the Pareto-optimal frontier of the solar power trace used in the previous examples (Dataset 1) and another data trace from the same location (a different year) (Dataset 2), using operating Strategy 2. Fig. 7b compares the Pareto-optimal frontier of the original trace (Location 1) and another location (Location 2), using operating Strategy 2. Fig. 7 shows that the Pareto-optimal frontier is sensitive to both temporal and spatial variations. This is due to the fact that we design the system for the worst case scenario, i.e., the system should meet the demand at all times. Replacing the input trace with another sample path creates a different worstcase scenario which might lead to a different Pareto frontier. In future work, we plan to extend our work to a probabilistic setting where the demand needs to be met almost surely, but not always.

\section{RELATED WORK}

A hybrid storage system comprising $N$ storage elements can combine the best features of each element. This allows it to outperform any single system element in terms of a given cost function, such as total investment, return on investment (ROI), system efficiency, and system lifetime. However, the design of an effective HESS is difficult, due to three coupled design factors: the choice of the storage technologies, operating strategy, and storage sizing. Moreover, the optimal design of an HESS is highly application-dependent [1], [2], [3].

Recent papers have studied the role of HESS in different applications. These can be categorized into four classes. The first is source shaping, i.e., to reshape power sources or to smooth out the fluctuations of renewable energy (e.g., [6]). Second, demand shaping or load reshaping. Examples of this application are load leveling or peak shaving of datacenters [8]. Third, HESS can be also used for residential prosumers. For example, HESS can be used to minimize the daily electricity cost for residential prosumers with PV panels. Depending on the time of use pricing, the residential prosumers can use either their locally generated solar power or buy electricity from the grid. Storage devices allow household prosumers to optimally shift their power generation or their load demand in order to minimize their electricity bills. It is shown that an HESS composed of $\mathrm{PbA}$ and $\mathrm{Li}$-ion batteries achieves an annual ROI of up to $60 \%$ higher than a single-element $\mathrm{PbA}$ or lithium-ion system, given a fixed monetary budget [3], [9]. Finally, HESS can be used in electric vehicles [4], [5].

The optimal choice of storage technologies for each application is governed by the energy and power efficiency of the storage technologies and the time scales of that application. Typically, an HESS combines a high energy efficient storage technology such as batteries with a high power efficient storage technology such as SCap. In [18] four HESS configurations have been considered for decentralized PV systems: powerto-heat/battery, power-to-heat/battery/hydrogen, SCap/battery, and battery/battery. For a given choice of storage technologies, the next problem is how to minimally size the underlying elements to adequately satisfy application requirements.

Prior wok in HESS operating strategies can be categorized into rule-based or optimization-based strategies [18]. Rulebased operating strategies are not extracted directly from solving the optimization problem [19]: Strategy 1 and Strategy 2 in this paper are examples of rule-based operating strategies. A slightly more complicated version of rule-based operating strategies are those with "thermostat" concept, which switch states whenever the energy level of the storage elements exceed or go below a predefined threshold. In contrast optimization-based operating strategies are extracted from the optimization solution [20]. They are either globally optimal and based on traces, or they are model predictive and real-time. The solution to the optimization problem in this paper finds the globally optimal operating strategy, which then inspires a simple rule-based operating strategy. Despite being impractical for real systems, globally optimal operating strategies are useful as benchmarks. Choosing a rule-based or a real-time (modelbased) operating strategy is a trade-off between complexity and performance.

Optimizing over all three design factors simultaneously is challenging and has not been studied well. Instead, one or two elements are assumed to be given and the design optimization is only over the remaining elements. For example, 
there are several papers studying the optimal control strategy for different applications [7], [4], [1], assuming the storage technology and sizes are given. Yang and Nehorai [11] jointly size an HESS and size PV panels for a limited choice of operating strategies (without charge migration) to minimize the initial investment cost and the maintenance cost. Note that disallowing charge migration greatly limits the choice of operating strategies and unclear at this point how much we lose by doing so. In [12], the optimal placement and sizing of a (single) storage technology is formulated to minimize the generation cost.

At this point, it appears that a full understanding of the performance and feasibility of an HESS over the spectrum of the design factors is limited to few applications and cost functions. Our work is a first attempt to account for all three important elements in the HESS design space. Finally, unlike all the existing work, our framework is applicable to a wide range of applications and the choice of storage technologies.

\section{CONCLUSION}

Each storage technology has its own power and energy characteristics and it appears that no single technology is likely to meet the requirements of all applications of storage. This motivates the use of hybrid energy storage systems (HESS) to combine the best features of multiple storage technologies. The performance of an HESS depends on three coupled factors: the choice of storage technologies, their sizes, and the charging/discharging operating strategy. The interplay between these three factors makes the optimal design of an HESS a challenging open problem. In this paper, we formulate the optimal design of an HESS, computing the optimal sizing and operating strategy for given set of storage technologies. Iterating over different choices of storage technologies, this allows us to explore the entire design space for the first time. Our problem formulation returns the Pareto-optimal frontier of the sizes of the underlying storage elements. This frontier can be used to find the optimal storage sizing for any application with a non-decreasing objective function in the vector of storage sizes. We prove that our original non-linear problem formulation can be converted to an LP, substantially reducing the computation time. Our problem formulation can be applied to a large set of applications and storage elements. We use this formulation for two case studies. We also find practical (i.e., trace independent and myopic) operating strategies which perform close to the optimal trace-dependent one. Finally, using real traces, we find several interesting engineering insights.

\section{REFERENCES}

[1] M. Pedram, N. Chang, Y. Kim, and Y. Wang, "Hybrid electrical energy storage systems," in Proceedings of the 16th ACM/IEEE International Symposium on Low Power Electronics and Design, pp. 363-368, 2010.

[2] S. Vosen and J. Keller, "Hybrid energy storage systems for stand-alone electric power systems: optimization of system performance and cost through control strategies," International Journal of Hydrogen Energy, vol. 24, no. 12, pp. 1139 - 1156, 1999.

[3] Y. Wang, X. Lin, M. Pedram, S. Park, and N. Chang, "Optimal control of a grid-connected hybrid electrical energy storage system for homes," in Automation and Test in Europe Proceedings of the Conference on Design, pp. 881 - 886, 2013.
[4] P. Thounthong, S. Ral, and B. Davat, "Energy management of fuel cell/battery/supercapacitor hybrid power source for vehicle applications," Journal of Power Sources, vol. 193, no. 1, pp. 376 - 385, 2009.

[5] S. Lu, K. Corzine, and M. Ferdowsi, "A new battery/ultracapacitor energy storage system design and its motor drive integration for hybrid electric vehicles," IEEE Transactions on Vehicular Technology, vol. 56, pp. 1516-1523, July 2007.

[6] S. Teleke, M. Baran, S. Bhattacharya, and A. Huang, "Optimal control of battery energy storage for wind farm dispatching," IEEE Transactions on Energy Conversion, vol. 25, pp. 787-794, September 2010.

[7] P. Thounthong, V. Chunkag, P. Sethakul, S. Sikkabut, S. Pierfederici, and B. Davat, "Energy management of fuel cell/solar cell/supercapacitor hybrid power source," Journal of Power Sources, vol. 196, no. 1, pp. 313 $-324,2011$.

[8] D. Wang, C. Ren, A. Sivasubramaniam, B. Urgaonkar, and H. Fathy, "Energy storage in datacenters: What, where, and how much?," in Proceedings of the ACM SIGMETRICS/PERFORMANCE, pp. 187-198, 2012.

[9] D. Zhu, Y. Wang, S. Yue, Q. Xie, M. Pedram, and N. Chang, "Maximizing return on investment of a grid-connected hybrid electrical energy storage system," in Design Automation Conference (ASP-DAC), 2013 18th Asia and South Pacific, pp. 638 - 643, Jan 2013.

[10] Y. Ghiassi-Farrokhfal, S. Keshav, and C. Rosenberg, "Toward a realistic performance analysis of storage systems in smart grids," IEEE Transactions on Smart Grid, vol. 6, pp. 402-410, Jan 2015.

[11] P. Yang and A. Nehorai, "Joint optimization of hybrid energy storage and generation capacity with renewable energy," IEEE Transactions on Smart Grid, vol. 5, no. 4, pp. 1566-1574, 2014.

[12] C. Thrampoulidis, S. Bose, and B. Hassibi, "Optimal placement of distributed energy storage in power networks," IEEE Transactions on Automatic Control, DOI: 10.1109/TAC.2015.2437527, 2015.

[13] A. Burke, "Ultracapacitors: why, how, and where is the technology," Journal of Power Sources, vol. 91, no. 1, pp. 37-50, 2000.

[14] http://batteryuniversity.com.

[15] E. M. Krieger, Effects of variability and rate on battery charge storage and lifespan. PhD thesis, Princeton University, Princeton, NJ, 2012.

[16] G. Ning, B. Haran, and B. N. Popov, "Capacity fade study of lithiumion batteries cycled at high discharge rates," Journal of Power Sources, vol. 117 , no. 12 , pp. $160-169,2003$.

[17] http://www.archive.arm.gov.

[18] T. Bocklisch, "Hybrid energy storage systems for renewable energy applications," Energy Procedia, vol. 73, pp. 103 - 111, 2015.

[19] K. Jin, X. Ruan, M. Yang, and M. Xu, "A hybrid fuel cell power system," IEEE Transactions on Industrial Electronics, vol. 56, no. 4, pp. 12121222, 2009.

[20] X. Zhang, C. C. Mi, A. Masrur, and D. Daniszewski, "Wavelettransform-based power management of hybrid vehicles with multiple on-board energy sources including fuel cell, battery and ultracapacitor,' Journal of Power Sources, vol. 185, no. 2, pp. 1533 - 1543, 2008.

\section{APPENDIX}

\section{A. 1: ProOF OF LEMMA 1}

Suppose that the starred values $\left(I_{i}^{*}, O_{i}^{*}, e_{i j}^{*}, J^{*}\right)$ for any $1 \leq i, j \leq N$ refer to a solution of the optimization problem (skipping $(k)$ in notation for convenience). While this solution does not necessarily satisfy the claim in the lemma, we can transform it to a new set of variables $\left(I_{i}^{\prime}, O_{i}^{\prime}, e_{i j}^{\prime}, J^{\prime}\right)$ for any $1 \leq i, j \leq N$ that is also a solution of the problem and yet satisfies the claim of the Lemma. The values of $\left(I_{i}^{\prime}, O_{i}^{\prime}, e_{i j}^{\prime}\right.$, $J^{\prime}$ ) are chosen in a way to maintain the values of $b_{i}$ for any $1 \leq i \leq N$ from Eq. (12h) unchanged (compared to those with the starred values) and satisfy all other constraints, so the objective function is not affected by this transformation.

To present how to compute $\left(I_{i}^{\prime}, O_{i}^{\prime}, e_{i j}^{\prime}, J^{\prime}\right)$, define $X_{i}$ such that

$$
X_{i}:=\eta_{i}^{c}\left(I_{i}^{*}+\sum_{j=1}^{N} e_{j i}^{*}\right)-\frac{1}{\eta_{i}^{d}}\left(O_{i}^{*}+\sum_{j=1}^{N} e_{i j}^{*}\right)
$$


From the energy content evolution formulations (Eq. (12h)), the sign of $X_{i}$ determines if the device $i$ is either in charging or discharging mode. To simplify notation, denote $N_{c h}$ and $N_{d c}$, respectively, the set of storage devices in charging and discharging mode (at time $k$ ). This is

$$
N_{c h}=\left\{i \mid X_{i} \geq 0\right\} ; \quad N_{d c}=\left\{i \mid X_{i}<0\right\}
$$

With this notation, set $e_{i j}^{\prime}$ to be

$e_{i j}^{\prime}=$

$\begin{cases}{\left[e_{i j}^{*}-e_{j i}^{*} /\left(\eta_{j}^{c} \eta_{j}^{d}\right)-Y_{i j}-Z_{i j}\right]_{+}} & \text {if } i \in N_{d c} \text { and } j \in N_{c h} \\ 0 & \text { Otherwise }\end{cases}$

where $[x]_{+}=\max (0, x)$ for any $x$. For any $j \in N_{c h}, Y_{i j}$ is given by

$$
Y_{i j}= \begin{cases}0 & \forall i \notin N_{d c} \\ A_{i j} \frac{B_{i j}}{\sum_{i \in N_{d c}} B_{i j}} & \forall i \in N_{d c}\end{cases}
$$

where

$$
\begin{aligned}
A_{i j} & :=\min \left(\sum_{i \in N_{d c}}\left[e_{i j}^{*}-e_{j i}^{*} /\left(\eta_{j}^{c} \eta_{j}^{d}\right)\right]_{+}, O_{j}^{*} /\left(\eta_{j}^{c} \eta_{j}^{d}\right)\right) \\
B_{i j} & :=\left[e_{i j}^{*}-e_{j i}^{*} /\left(\eta_{j}^{c} \eta_{j}^{d}\right)\right]_{+}
\end{aligned}
$$

Given the values of $Y_{i j}$, for any $i \in N_{d c}, Z_{i j}$ is computed as

$$
Z_{i j}= \begin{cases}0 & \forall j \notin N_{c h} \\ C_{i j} \frac{D_{i j}}{\sum_{j \in N_{c h}} D_{i j}} & \forall j \in N_{c h},\end{cases}
$$

where

$$
\begin{aligned}
& C_{i j}:=\max \left(0, \sum_{j \in N_{c h}}\left[e_{i j}^{*}-e_{j i}^{*} /\left(\eta_{j}^{c} \eta_{j}^{d}\right)-Y_{i j}\right]_{+}+\eta_{i}^{d} X_{i}\right) \\
& D_{i j}:=\left[e_{i j}^{*}-e_{j i}^{*} /\left(\eta_{j}^{c} \eta_{j}^{d}\right)-Y_{i j}\right]_{+}
\end{aligned}
$$

The role of $Y_{i j}$ and $Z_{i j}$ is to ensure that for any $j \in N_{c h}$

$$
\sum_{i \in N} e_{i j}^{\prime}=\sum_{i \in N_{d c}}\left[e_{i j}^{*}-\left(e_{j i}^{*}+O_{j}^{*}\right) /\left(\eta_{j}^{c} \eta_{j}^{d}\right)\right]_{+}
$$

unless $e_{i j}^{\prime}$ is bounded by the following for some $i \in N_{d c}$

$$
\sum_{j \in N} e_{i j}^{\prime} \leq-\eta_{i}^{d} X_{i} .
$$

in which case $e_{i j}^{\prime}$ for $j \in N$ will be curtailed to satisfy the above constraint.

Having the values of $e_{i j}^{\prime}$ for any $1 \leq i, j \leq N$, set the values of $I_{i}^{\prime}, O_{i}^{\prime}$, and $J^{\prime}$ accordingly as follows

$$
\begin{aligned}
I_{i}^{\prime} & =\left[X_{i} / \eta_{i}^{c}-\sum_{j=1}^{N} e_{j i}^{\prime}\right]_{+} \\
O_{i}^{\prime} & =\left[-\eta_{i}^{d} X_{i}-\sum_{j=1}^{N} e_{j i}^{\prime}\right]_{+} \\
J^{\prime} & =\left[J^{*}-\sum_{j=1}^{N} O_{j}^{\prime}+\sum_{j=1}^{N} O_{j}^{*}\right]_{+} .
\end{aligned}
$$

This choice of $J^{\prime}$ ensures that $J^{\prime} \geq 0$ and moreover, Eq. (12c) with the modified variables holds (knowing that it holds for the starred values). In addition, the specific choices of the variables in the above four cases guarantee that $I_{i}^{\prime}, O_{i}^{\prime}$, $e_{j i}^{\prime} \geq 0$ for any $1 \leq i, j \leq N$. These choices also ensure that $b_{i}$ is unchanged. Therefore, we only need to show that the constraints in Eq. (12d) to Eq. (12f) hold in all cases.

With the choice of $J^{\prime}$ in Eq. (31), to prove that Eq. (12d) for the modified variables hold, we have to show that both of the following constraints hold:

$$
\begin{gathered}
\sum_{i=1}^{N} I_{i}^{\prime} \leq S \\
\sum_{i=1}^{N} I_{i}^{\prime}-\sum_{i=1}^{N} O_{i}^{\prime}+\sum_{i=1}^{N} O_{i}^{*} \leq S-J^{*}
\end{gathered}
$$

From Eq. (12d) for the optimal values, we know that

$$
\sum_{i=1}^{N} I_{i}^{*} \leq S-J^{*} .
$$

Therefore, sufficient conditions for Eqs. (32)-(33) to hold are

$$
\begin{gathered}
\sum_{i=1}^{N} I_{i}^{\prime} \leq \sum_{i=1}^{N} I_{i}^{*} \\
\sum_{i=1}^{N}\left(I_{i}^{\prime}-O_{i}^{\prime}\right) \leq \sum_{i=1}^{N}\left(I_{i}^{*}-O_{i}^{*}\right)
\end{gathered}
$$

In summary, to prove the lemma, it is sufficient to show that with the choices in Eq. (20) to Eq. (31) satisfy the four constraints in Eq. (12e) to Eq. (12f) and Eqs. (35)-(36). It can be verified by replacing the values of $\left(I_{i}^{\prime}, O_{i}^{\prime}, e_{i j}^{\prime}, J^{\prime}\right)$ for any $1 \leq i, j \leq N$ in those four constraints.

\section{A.2: FORMULATING THE ENERGY CONTENT EVOLUTION OF SCAPS}

The terminal voltage of the SCap must not exceed an upper bound threshold $V_{\max }$ to ensure that it is safely operating. For a Scap with capacity $C$ and maximum voltage $V_{\max }$, the maximum amount of energy that can be stored in the device, is equal to $B_{2}=\frac{1}{2} C V_{\max }^{2}$. The input power $P_{i o}$ is fixed during each time slot; $P_{i o}(k)$ denotes the input power during the $k$ 'th time slot or in the time interval $[k \delta,(k+1) \delta]$. Let $b_{2}(k)$ denote the energy content of the SCap at the beginning of time slot $k$. Using the energy conservation law, the stored energy in the SCap evolves according to the following differential equation:

$$
P_{i o}(k)=\frac{V_{c}(t)^{2}}{R_{d}}+\frac{d b_{2}(t)}{d t} ; \quad \forall t \in[k \delta,(k+1) \delta]
$$

where $V_{c}(t)$ denotes the SCap's voltage at time instant $t$. Inserting $S(t)=\frac{1}{2} C V_{c}(t)^{2}$ in Eq. (37), yields

$$
P_{i o}(k)=\frac{2 b_{2}(t)}{R_{d} C}+\frac{d S(t)}{d t} ; \quad \forall t \in[k \delta,(k+1) \delta]
$$


The solution to Eq. (38) is as follows:

$\forall t \in[k \delta,(k+1) \delta]:$

$b(t)=\left(\frac{C}{2} P_{i o}(k) R_{d}\right)+\left(b(k)-\frac{C}{2} P_{i o}(k) R_{d}\right) e^{-\left(\frac{2(t-k \delta)}{R_{d} C}\right)} ;$

Therefore, the energy stored at $t=(k+1) \delta$ is equal to

$b_{2}(k+1)=\left(\frac{C}{2} P_{i o}(k) R_{d}\right)+\left(b(k)-\frac{C}{2} P_{i o}(k) R_{d}\right) e^{-\left(\frac{2 \delta}{R_{d} C}\right)}$.

Using the first order approximation of $e^{-\left(\frac{2 \delta}{R_{d} C}\right)}$, Eq. (40) is reduced to:

$$
b_{2}(k+1) \approx \Gamma b_{2}(k)+P_{i o}(k) \delta,
$$

where $\Gamma=\left(1-\frac{2 \delta}{R_{d} C}\right)$ denotes the self-discharge efficiency of the storage within a time slot. Thus, we have transformed the non-linear energy content evolution of a SCap to a linear one with a first order approximation.

Note that $P_{i o}$ represents both the input $\left(P_{i o}>0\right)$ and the output power $\left(P_{i o}<0\right)$ to the SCap. Thus, Eq. (41) can be rewritten as

$$
b_{2}(k+1) \approx \Gamma b_{2}(k)+S_{2}(k) \delta-D_{2}(t) \delta,
$$

where $S_{2}$ and $D_{2}$ are, respectively, the input and output power to the SCap. In addition to Eq. (42), the values of $S_{2}$ and $D_{2}$ must ensure that the following constraint holds

$$
0 \leq b_{2}(k) \leq B_{2}
$$

or

$$
0 \leq b_{2}(k) \leq 1 / 2 C V_{\max }^{2}
$$

The values of $C, R_{d}$, and $V_{\max }$ can be collected from the data sheet of SCaps.

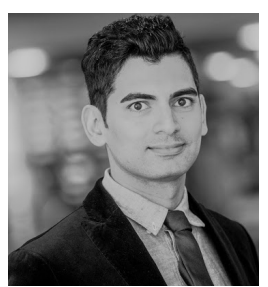

Yashar Ghiassi-Farrokhfal (S'09-M'10) is an Assistant Professor at the Department of Technology and Operations Management at Rotterdam School of Management, Erasmus University.

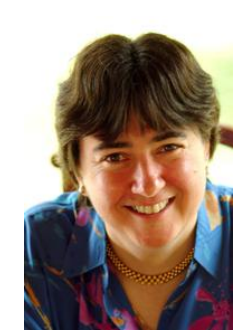

Catherine Rosenberg (M'89-SM'96-F'11) is a Professor, Canadian Research Chair, and Fellow of the Canadian Academy of Engineering with the Department of Electrical and Computer Engineering, University of Waterloo, Waterloo, ON, Canada.

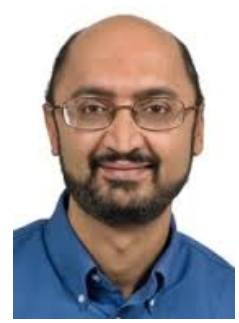

Srinivasan Keshav (M'99) is a Professor, ACM Fellow, and Cisco Systems Research Chair in Smart Grid with the Cheriton School of Computer Science, University of Waterloo, Waterloo, ON, Canada.

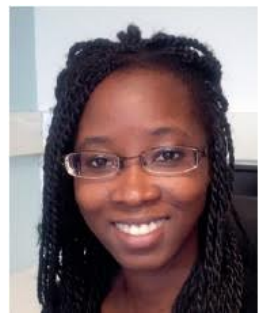

Marie - Benedicte Adjaho received her Master of Engineering (M.Eng.) from Ecole Nationale Superieure des Telecommunications de Bretagne, Brest, France in September 2015. She has been working in Songhai Energy Limited, Porto Novo, Benin as of October 2015. 